TRANSACTIONS OF THE

AMERICAN MATHEMATICAL SOCIETY

Volume 348 , Number 11, November 1996

\title{
EIGENVALUE ASYMPTOTICS AND EXPONENTIAL DECAY OF EIGENFUNCTIONS FOR SCHRÖDINGER OPERATORS WITH MAGNETIC FIELDS
}

\author{
ZHONGWEI SHEN
}

ABSTRACT. We consider the Schrödinger operator with magnetic field,

$$
H=\left(\frac{1}{i} \nabla-\vec{a}(x)\right)^{2}+V(x) \quad \text { in } \mathbb{R}^{n} .
$$

Assuming that $V \geq 0$ and $|\operatorname{curl} \vec{a}|+V+1$ is locally in certain reverse Hölder class, we study the eigenvalue asymptotics and exponential decay of eigenfunctions.

\section{INTRODUCTION}

Consider the Schrödinger operator with magnetic field

$$
H=H(\vec{a}, V)=\left(\frac{1}{i} \nabla-\vec{a}(x)\right)^{2}+V(x) \quad \text { in } \mathbb{R}^{n}, n \geq 3,
$$

where $i=\sqrt{-1}, \quad V: \mathbb{R}^{n} \rightarrow \mathbb{R}$ is the electric (scalar) potential and $\vec{a}: \mathbb{R}^{n} \rightarrow \mathbb{R}^{n}$ is the magnetic (vector) potential. Under some natural conditions on $\vec{a}$ and $V$, $H$ admits a self-adjoint realization, which is still denoted by $H$, in $L^{2}\left(\mathbb{R}^{n}\right)$. In this paper we shall study its eigenvalue asymptotics and exponential decay of its eigenfunctions.

Let $N(\lambda, H)$ denote the number of eigenvalues (counting multiplicity) of $H$ smaller than $\lambda$ (or the dimension of the spectral projection for $H$ corresponding to $(-\infty, \lambda))$. In the case $\vec{a}(x) \equiv \overrightarrow{0}$, the following phase-space volume estimate due to Cwickel-Lieb-Rosenbljum [Si1, Theorem 9.3, p. 95] is classical:

$$
N(\lambda,-\Delta+V) \leq c_{n}\left|\left\{(x, \xi) \in \mathbb{R}^{n} \times \mathbb{R}^{n}:|\xi|^{2}+V(x)<\lambda\right\}\right|
$$

where $c_{n}$ is a constant depending on $n$. On the other hand, there exist some simple degenerate potentials $V(x)$ (i.e., potentials which do not tend to infinity in all direction as $|x| \rightarrow \infty$, e.g., $\left.V(x)=V\left(x_{1}, x_{2}, \ldots, x_{n}\right)=x_{1}^{2} x_{2}^{2} \ldots x_{n}^{2}\right)$ for which, the right-hand side of $(0.2)$ is infinite and, nevertheless, $-\Delta+V(x)$ has a discrete spectrum. Furthermore, in the case where $\vec{a}(x) \not \equiv \overrightarrow{0}$ and $V(x) \equiv 0$, the phase-space volume of the set where the symbol of $H$ is less than $\lambda$,

$$
\left|\left\{(x, \xi) \in \mathbb{R}^{n} \times \mathbb{R}^{n}: \sum_{j=1}^{n}\left|\xi_{j}-a_{j}(x)\right|^{2}<\lambda\right\}\right|,
$$

Received by the editors May 19, 1995.

1991 Mathematics Subject Classification. Primary 35P20, 35J10.

Key words and phrases. Eigenvalue asymptotics, Schrödinger operator, reverse Hölder class. 
is always infinite for any $\vec{a}(x)=\left(a_{1}(x), a_{2}(x), \cdots, a_{n}(x)\right)$ and $\lambda>0$. Recently, there has been considerable interest in this kind of non-classical eigenvalue asymptotics. See e.g. [F] [Gu] [HMo] [I] [Ma] [MoN] [R] [Si2] [So] [T].

The main purpose of this paper is to introduce a class of potentials for which we can estimate $N(\lambda, H)$ in the spirit of $(0.2)$ both from above and below.

More precisely, we consider potentials which locally belong to the reverse Hölder class.

Definition 0.3. Suppose $W \in L_{\mathrm{loc}}^{p}\left(\mathbb{R}^{n}\right)(1<p<\infty)$ and $W \geq 0$ a.e. on $\mathbb{R}^{n}$. We say $W \in(R H)_{p}$, loc if there exists $C_{0} \geq 1$ such that

$$
\left(\frac{1}{|B(x, r)|} \int_{B(x, r)} W^{p}(y) d y\right)^{\frac{1}{p}} \leq C_{0} \cdot \frac{1}{|B(x, r)|} \int_{B(x, r)} W(y) d y
$$

for every $x \in \mathbb{R}^{n}$ and $0<r \leq 1$, where $B(x, r)$ denotes the ball centered at $x$ with radius $r$. If (0.4) holds for $0<r<\infty$, we say $W \in(R H)_{p}$.

The reverse Hölder class $(R H)_{p}$ (also known as the $B_{p}$ weights) was introduced by Gehring $[\mathrm{Ge}]$ and Muckenhoupt $[\mathrm{Mu}]$ in the study of quasi-conformal mapping and weighted norm inequalities, respectively. It is not hard to see that, if $P(x)$ is a polynomial and $\alpha>0$, then $W=|P(x)|^{\alpha} \in(R H)_{p}$ for any $p>1$. Also, note that $W(x)=e^{|x|}$ is in $(R H)_{p, \text { loc }}$, but not in $(R H)_{p}$. Hence,

$$
(R H)_{p} \subsetneq(R H)_{p, \text { loc }}
$$

Definition 0.5. For $x \in \mathbb{R}^{n}$, the function $m(x, W)$ is defined by

$$
\frac{1}{m(x, W)}=\sup \left\{r>0: \frac{r^{2}}{|B(x, r)|} \int_{B(x, r)} W(y) d y \leq 1\right\} .
$$

The function $m(x, W)$ is closely related to the uncertainty principle. If $W=$ $|P(x)|^{\alpha}$ where $\alpha>0$ and $P(x)$ is a polynomial of degree $k$, it is shown in [Sh2] that

$$
m(x, W) \approx \sum_{|\beta| \leq k}\left|\partial_{x}^{\beta} P(x)\right|^{\frac{\alpha}{\alpha|\beta|+2}} .
$$

Let

$$
B(x)=\operatorname{curl} \vec{a}(x)=\left(b_{j k}(x)\right)_{1 \leq j, k \leq n}
$$

be the magnetic field generated by $\vec{a}(x)$ where

$$
b_{j k}=\frac{\partial a_{j}}{\partial x_{k}}-\frac{\partial a_{k}}{\partial x_{j}}
$$

The following is one of main results in the paper.

Theorem 0.9. Suppose $\vec{a} \in C^{2}\left(\mathbb{R}^{n}\right), V \in L_{\text {loc }}^{\frac{n}{2}}\left(\mathbb{R}^{n}\right)$ and $V \geq 0$ a.e. on $\mathbb{R}^{n}$. Also assume that

$$
\left\{\begin{array}{l}
|B|+V+1 \in(R H)_{\frac{n}{2}}, \text { loc } \\
|B(x)| \leq C_{1}\{m(x,|B|+V+1)\}^{2} \\
|\nabla B(x)| \leq C_{2}\{m(x,|B|+V+1)\}^{3}
\end{array}\right.
$$


where $|B|=|B(x)|=\sum_{j, k}\left|b_{j k}(x)\right|$. Then, there exist constants $C>0$ and $c>0$, which depend only on $n$ and the constants $C_{0}, C_{1}, C_{2}$ in (0.4) and (0.10), such that, for $\lambda \geq C$,

$$
\left\{\begin{array}{l}
N(\lambda, H(\vec{a}, V)) \leq C\left|\left\{(x, \xi) \in \mathbb{R}^{n} \times \mathbb{R}^{n}:|\xi|^{2}+\{c m(x,|B|+V+1)\}^{2}<\lambda\right\}\right|, \\
N(\lambda, H(\vec{a}, V)) \geq c\left|\left\{(x, \xi) \in \mathbb{R}^{n} \times \mathbb{R}^{n}:|\xi|^{2}+\{C m(x,|B|+V+1)\}^{2}<\lambda\right\}\right| .
\end{array}\right.
$$

Theorem 0.9 allows one to estimate the leading power of $N(\lambda, H(\vec{a}, V))$ in many cases for degenerate potentials $V(x)$, as well as degenerate magnetic fields $B(x)$. In particular, we have

Corollary 0.11. Suppose $\vec{a}$ and $V$ satisfy the same hypothesis of Theorem 0.9. Then $H(\vec{a}, V)$ has a discrete spectrum if and only if

$$
\lim _{|x| \rightarrow \infty} m(x,|B|+V)=\infty .
$$

Our estimates in Theorem 0.9 are closely related to that in [MoN], where similar results are obtained for potentials which are polynomials or behave like polynomials. Indeed, in $[\mathrm{MoN}]$, it is assumed that

$$
B \in C^{k+1}\left(\mathbb{R}^{n}\right), \quad V \in C^{k+2}\left(\mathbb{R}^{n}\right), \quad V \geq 0,
$$

and

$$
\sum_{|\beta|=k+1}\left|\partial_{x}^{\beta} B(x)\right|+\sum_{|\beta|=k+2}\left|\partial_{x}^{\beta} V(x)\right| \leq C\{1+M(x)\}
$$

where

$$
M(x)=\sum_{|\beta| \leq k}\left|\partial_{x}^{\beta} B(x)\right|+\sum_{|\beta| \leq k+1}\left|\partial_{x}^{\beta} V(x)\right| .
$$

Using the Taylor formula, one can show that, if $B$ and $V$ satisfy (0.12), then

$$
|B|+V+1 \in(R H)_{p} \text {, loc for all } p, 1<p<\infty .
$$

In fact, (0.12) implies that, for $0<r \leq 1$,

$$
\sup _{y \in B(x, r)}\{|B(y)|+V(y)+1\} \leq \frac{C}{|B(x, r)|} \int_{B(x, r)}\{|B(y)|+V(y)+1\} d y
$$

and

$$
m(x,|B|+V+1) \approx \sum_{|\beta| \leq k}\left|\partial_{x}^{\beta} B(x)\right|^{\frac{1}{|\beta|+2}}+\sum_{|\beta| \leq k+1}\left|\partial_{x}^{\beta} V(x)\right|^{\frac{1}{\beta \beta+2}}+1 .
$$

Clearly, condition (0.10) follows from (0.13) and (0.14). Thus, our Theorem 0.9 generalizes the results in $[\mathrm{MoN}]$. The main interest here is that our assumption $(0.10)$ is much weaker and only requires minimal smoothness condition for the magnetic field $B(x)$ and no smoothness condition for the electric potential $V(x)$.

The proof of Theorem 0.9 relies on the following estimate:

$$
\begin{aligned}
& \int_{\mathbb{R}^{n}}|m(x,|B|+V) u(x)|^{2} d x \\
& \leq C\left\{\sum_{j=1}^{n} \int_{\mathbb{R}^{n}}\left|\left(\frac{1}{i} \frac{\partial}{\partial x_{j}}-a_{j}(x)\right) u\right|^{2} d x+\int_{\mathbb{R}^{n}}(V(x)+1)|u(x)|^{2} d x\right\} .
\end{aligned}
$$


Estimates similar (0.15) have been established for many special cases [F] [HMo] [HN1] [MoN] [Sh1]. In particular, for the case where $\vec{a}(x) \equiv \overrightarrow{0}$ and $V$ is a nonnegative polynomial, (0.15) follows from [F, Main Lemma, p. 146]. The estimate was generalized to the case $\vec{a} \equiv \overrightarrow{0}$ and $V \in(R H)_{n / 2}$ in [Sh1].

In the case when the magnetic potential $\vec{a}(x)$ is present, $(0.15)$ was proved for polynomial potentials in [HN1] and for potentials which satisfy (0.12) in [HMo] $[\mathrm{MoN}]$. This was done by using the technique of higher-order commutators, which could not be applied to our case due to the lack of smoothness on $\vec{a}$ and $V$. Instead, we shall adapt the idea of Fefferman-Phong $[\mathrm{F}]$ and exploit the uncertainty principle.

Theorem 0.9 follows from estimate $(0.15)$ and the use of the minimax principle, by constructing certain subspaces of $L^{2}\left(\mathbb{R}^{n}\right)$.

In this paper we shall also investigate the exponential decay of eigenfunctions of $H(\vec{a}, V)$ under the assumption of Theorem 0.9.

Following [HN2], we introduce the distance function $d(x, y)$ for the modified Agmon metric

$$
\begin{gathered}
d s^{2}=m(x,|B|+V+1) d x^{2} \\
d(x, y)=\inf \left\{\int_{0}^{1} m(\gamma(t),|B|+V+1)\left|\gamma^{\prime}(t)\right| d t:\right. \\
\left.\gamma:[0,1] \rightarrow \mathbb{R}^{n}, \gamma(0)=x \text { and } \gamma(1)=y\right\} .
\end{gathered}
$$

For $\lambda>0$, let

$$
E_{\lambda}=\left\{x \in \mathbb{R}^{n}: m(x,|B|+V+1) \leq \sqrt{\lambda}\right\}
$$

and

$$
d_{\lambda}(x)=d\left(x, E_{\lambda}\right)=\inf \left\{d(x, y): y \in E_{\lambda}\right\} .
$$

Theorem 0.20. Under the same assumption of Theorem 0.9 , there exist $C>0$ and $\varepsilon>0$ depending only on $n$ and $C_{0}, C_{1}, C_{2}$ in (0.4) and (0.10), such that, if $u \in L^{2}\left(\mathbb{R}^{n}\right), H(\vec{a}, V) u \in L^{2}\left(\mathbb{R}^{n}\right)$ and

$$
H(\vec{a}, V) u=\lambda u, \lambda \geq C,
$$

then

$$
|u(x)| \leq C \lambda^{\frac{n}{2}} e^{-\varepsilon d_{C \lambda}(x)}\|u\|_{2} .
$$

Remark 0.21. If, in addition, we assume in Theorem 0.20 that

$$
\lim _{|x| \rightarrow \infty} m(x,|B|+V)=\infty,
$$

then $d_{C \lambda}(x) \leq C_{\lambda}+d(x)$ where $d(x)=d(x, 0)$. It follows that

$$
|u(x)| \leq C_{\lambda} e^{-\varepsilon d(x)}\|u\|_{2} .
$$

Theorem 0.20 generalizes the results in [HN2], where a similar estimate was proved for polynomial electrical and magnetic potentials. The approach in [HN2] is based on a higher-order estimate of $H^{m}$, obtained by the techniques of Lie algebra. In our case, the definition of $H^{m}$ is even problematic. Here we shall rely on the estimate (0.15) and some $L^{\infty}$-estimates of $H(\vec{a}, V)$. 
The paper is organized as follows. In Section 1 we study the properties of the auxiliary function $m(x, W)$ defined in (0.5) and establish a simple form of Fefferman-Phong type estimate for the magnetic Schrödinger operator (Theorem 1.9). Estimate (0.15) is proved in Section 2 (Theorem 2.7). The proof of Theorem 0.9 is given in Section 3. Finally, we prove Theorem 0.20 in Section 4 .

Throughout this paper, unless otherwise indicated, we will use $C$ and $c$ to denote positive constants, which depend at most on the dimension $n$ and the constants in (0.4) and (0.10). By $A \approx B$, we mean that there exist constants $C>0$ and $c>0$, such that $c \leq A / B \leq C$.

\section{Preliminaries}

In this section we shall study the properties of the auxiliary function $m(x, W)$ defined in (0.5) and establish a simple form of Fefferman-Phong type estimate for the magnetic Schrödinger operator (Theorem 1.9).

Recall that we say $W \in(R H)_{p}$ for some $p>1$ if there exists $C \geq 1$ such that

$$
\left(\frac{1}{|B|} \int_{B} W^{p}(x) d x\right)^{\frac{1}{p}} \leq C \cdot \frac{1}{|B|} \int_{B} W(x) d x
$$

for every ball $B$ in $\mathbb{R}^{n}$. It is well known that, if $W \in(R H)_{p}$, then $W \in(R H)_{p+\varepsilon}$ for some $\varepsilon>0$ which depends only on $n$ and the constant in (1.1). Also, if $W \in$ $(R H)_{p}(p>1)$, then $d \mu=W d x$ is a doubling measure, i.e.,

$$
\int_{B(x, r)} W(y) d y \leq C \int_{B\left(x, \frac{r}{2}\right)} W(y) d y .
$$

In fact, $W d x$ is an $A_{\infty}$ weight. As a consequence, there exists $c>0$ such that

$$
\left|\left\{x \in B: W(x) \geq \frac{c}{|B|} \int_{B} W(y) d y\right\}\right| \geq c|B| .
$$

See [St, Chapter V].

Similar properties hold for functions in $(R H)_{p}$, loc if we require that the radius of the ball is less than one.

Proposition 1.4. Suppose $W \in(R H)_{p}$, loc for some $p>1$. Then

(a) $W \in(R H)_{p+\varepsilon, \text { loc }}$, for some $\varepsilon>0$,

(b) (1.2) and (1.3) hold for any ball $B(x, r)$ with $r \leq 1$.

The proof is exactly the same as in the case of $(R H)_{p}$, hence omitted. We refer readers to [St, Chapter V] for references on $(R H)_{p}$.

Lemma 1.5. Suppose $W \in(R H)_{n / 2, \text { loc }}$ and $W(x) \geq 1$ a.e. on $\mathbb{R}^{n}$. Then

(a) $1 \leq m(x, W)<\infty$ for every $x \in \mathbb{R}^{n}$,

(b) if $|x-y|<\frac{1}{m(x, W)}$, then

$$
m(x, W) \approx m(y, W),
$$

(c) if $|x-y| \leq 1$, then

$$
\left\{\begin{array}{l}
m(y, W) \leq C\{1+|x-y| m(x, W)\}^{k_{0}} m(x, W), \\
m(y, W) \geq \frac{c m(x, W)}{\{1+|x-y| m(x, V)\}^{k_{0} /\left(k_{0}+1\right)}}
\end{array}\right.
$$

for some $k_{0}=k_{0}\left(n, C_{0}\right)>0$. 
Proof. Since $W \in(R H)_{n / 2 \text {, loc }}$, by part (a) of Proposition 1.4, $W \in(R H)_{p}$, loc for some $p>n / 2$. Thus, by Hölder inequality, for $0<r<R \leq 1$,

$$
\begin{aligned}
\frac{1}{|B(x, r)|} \int_{B(x, r)} W(y) d y & \leq\left(\frac{1}{|B(x, r)|} \int_{B(x, r)} W^{p}(y) d y\right)^{1 / p} \\
& \leq\left(\frac{R}{r}\right)^{n / p}\left(\frac{1}{|B(x, R)|} \int_{B(x, R)} W^{p}(y) d y\right)^{1 / p} \\
& \leq C\left(\frac{R}{r}\right)^{n / p} \cdot \frac{1}{|B(x, R)|} \int_{B(x, R)} W(y) d y
\end{aligned}
$$

where we have used (0.4) in the last inequality.

It follows that

$$
\frac{r^{2}}{|B(x, r)|} \int_{B(x, r)} W(y) d y \leq C\left(\frac{r}{R}\right)^{2-(n / p)} \cdot \frac{R^{2}}{|B(x, R)|} \int_{B(x, R)} W(y) d y .
$$

Hence,

$$
\lim _{r \rightarrow 0} \frac{r^{2}}{|B(x, r)|} \int_{B(x, r)} W(y) d y=0 \quad \text { for every } x \in \mathbb{R}^{n}
$$

since $2-(n / p)>0$. This implies that $m(x, W)<\infty$ for every $x \in \mathbb{R}^{n}$ by definition (0.5). Since $W \geq 1$ a.e., it is easy to see that $m(x, W) \geq m(x, 1)=1$. Part (a) is proved.

To see part (b), let $r=\{m(x, V)\}^{-1}$. Since $d \mu=W d x$ is locally a doubling measure and $|x-y| \leq\{m(x, V)\}^{-1} \leq 1$, we have

$$
\frac{r^{2}}{|B(y, r)|} \int_{B(y, r)} W(z) d z \approx \frac{r^{2}}{|B(x, r)|} \int_{B(x, r)} W(z) d z=1 .
$$

Hence, by (1.6), it is not hard to see that

$$
\frac{1}{m(y, W)} \approx r
$$

Part (b) follows.

Part (c) also follows from the fact that $W d x$ is a doubling measure on balls with radius less than one. In the case $W \in(R H)_{n / 2}$, similar estimates were proved in [Sh1, Lemma 1.4] for all $x, y \in \mathbb{R}^{n}$. Since $|x-y| \leq 1$ and $\{m(x, V)\}^{-1} \leq 1$, the same argument as in [Sh1] may be carried out without any modification. We omit the details.

Let

$$
L_{j}=\frac{1}{i} \frac{\partial}{\partial x_{j}}-a_{j}(x), \quad 1 \leq j \leq n,
$$

where $a_{j} \in L_{\text {loc }}^{2}\left(\mathbb{R}^{n}\right)$ is a real-valued function.

The following lemma is very important in this paper. In the case $a_{j}(x) \equiv 0$, it is the Main Lemma in [F, p. 146].

Lemma 1.8. Suppose $V \in L_{\text {loc }}^{1}\left(\mathbb{R}^{n}\right), V \geq 0$ and

$$
\left|\left\{x \in B\left(x_{0}, r\right): V(x) \geq \frac{c_{0}}{r^{2}}\right\}\right| \geq c_{0} r^{n}
$$


for some $x_{0} \in \mathbb{R}^{n}, r>0$ and $c_{0}>0$. Then there exists a constant $C>0$ depending on $n$ and $c_{0}$, such that, for every $u \in C^{1}\left(\mathbb{R}^{n}\right)$,

$$
\frac{1}{r^{2}} \int_{B\left(x_{0}, r\right)}|u(x)|^{2} d x \leq C\left\{\int_{B\left(x_{0}, r\right)}|L u|^{2} d x+\int_{B\left(x_{0}, r\right)}|u(x)|^{2} V(x) d x\right\}
$$

where $|L u|^{2}=\sum_{j=1}^{n}\left|L_{j} u\right|^{2}$.

Proof. Let $u_{\varepsilon}=\sqrt{\varepsilon^{2}+|u|^{2}}$. It follows from $u_{\varepsilon}^{2}=\varepsilon^{2}+u \bar{u}$ that

$$
\begin{aligned}
2 u_{\varepsilon} \frac{\partial u_{\varepsilon}}{\partial x_{j}} & =\frac{\partial u}{\partial x_{j}} \cdot \bar{u}+u \cdot \frac{\partial \bar{u}}{\partial x_{j}} \\
& =i\left(\frac{1}{i} \frac{\partial u}{\partial x_{j}}-a_{j} u\right) \bar{u}-i u \overline{\left(\frac{1}{i} \frac{\partial u}{\partial x_{j}}-a_{j} u\right)} \\
& =i L_{j} u \cdot \bar{u}-i u \cdot \overline{L_{j} u} .
\end{aligned}
$$

Thus,

$$
u_{\varepsilon}\left|\frac{\partial u_{\varepsilon}}{\partial x_{j}}\right| \leq|u|\left|L_{j} u\right|
$$

Hence,

$$
\left|\frac{\partial u_{\varepsilon}}{\partial x_{j}}\right| \leq\left|L_{j} u\right|
$$

since $|u| \leq u_{\varepsilon}$.

Now, by the Poincaré inequality, for $y \in B=B\left(x_{0}, r\right)$,

$$
\int_{B}\left|u_{\varepsilon}(x)-u_{\varepsilon}(y)\right|^{2} d x \leq C r^{2} \sum_{j=1}^{n} \int_{B}\left|\frac{\partial u_{\varepsilon}}{\partial x_{j}}\right|^{2} d x \leq C r^{2} \sum_{j=1}^{n} \int_{B}\left|L_{j} u\right|^{2} d x .
$$

Let $\varepsilon \rightarrow 0$; by Fatou's Lemma we have

$$
\int_{B}|| u(x)|-| u(y)||^{2} d x \leq C r^{2} \int_{B}|L u|^{2} d x .
$$

Integrating in $y$ over $B$, we get

$$
\frac{1}{r^{n+2}} \int_{B} \int_{B}|| u(x)|-| u(y)||^{2} d y d x \leq C \int_{B}|L u|^{2} d x .
$$

This, together with the identity

$$
\frac{1}{r^{n}} \int_{B} \int_{B} V(y)|u(y)|^{2} d y d x=C \int_{B} V(x)|u(x)|^{2} d x,
$$

implies that

$$
\begin{aligned}
& \frac{1}{r^{n}} \int_{B} \int_{B}\left\{\left.\frac{c_{0}}{r^{2}}|| u(x)|-| u(y)\right|^{2}+V(y)|u(y)|^{2}\right\} d y d x \\
& \leq C\left\{\int_{B}|L u|^{2} d x+\int_{B} V(x)|u(x)|^{2} d x\right\} .
\end{aligned}
$$


Hence,

$$
\begin{aligned}
\frac{1}{r^{n}} \int_{B}|u(x)|^{2} d x & \cdot \int_{B} \min \left\{V(y), \frac{c_{0}}{r^{2}}\right\} d y \\
& \leq C\left\{\int_{B}|L u|^{2} d x+\int_{B} V(x)|u|^{2} d x\right\} .
\end{aligned}
$$

The lemma then follows from the assumption

$$
\left|\left\{y \in B: V(y) \geq \frac{c_{0}}{r^{2}}\right\}\right| \geq c_{0}|B| .
$$

We now prove a simple version of the Fefferman-Phong type estimate for the magnetic Schrödinger operator. The complete version, which takes the magnetic field into the account, will be given in the next section (Theorem 2.7).

Theorem 1.9. Suppose $V \in(R H)_{n / 2}$, loc. Then there exists a constant $C>0$ such that, for $u \in C_{0}^{1}\left(\mathbb{R}^{n}\right)$,

$$
\int_{\mathbb{R}^{n}}|m(x, V) u(x)|^{2} d x \leq C\left\{\int_{\mathbb{R}^{n}}|L u|^{2} d x+\int_{\mathbb{R}^{n}}(V(x)+1)|u(x)|^{2} d x\right\} .
$$

Proof. Replacing $V$ by $V+1$ if necessary, we may assume, without loss of generality, that $V \geq 1$.

Fix $x_{0} \in \mathbb{R}^{n}$, and let $r=\frac{1}{m\left(x_{0}, V\right)} \leq 1$. Then

$$
\frac{1}{\left|B\left(x_{0}, r\right)\right|} \int_{B\left(x_{0}, r\right)} V(y) d y=\frac{1}{r^{2}}
$$

It follows from part (b) of Proposition 1.4 that

$$
\left|\left\{x \in B\left(x_{0}, r\right): V(x) \geq \frac{c_{0}}{r^{2}}\right\}\right| \geq c_{0}|B| .
$$

Also, by part (b) of Lemma 1.5,

$$
m(x, V) \approx m\left(x_{0}, V\right)=\frac{1}{r} \quad \text { for every } x \in B\left(x_{0}, r\right) .
$$

Thus, by Lemma 1.8,

$$
\begin{aligned}
\int_{B\left(x_{0}, r\right)} & \{m(x, V)\}^{2+n}|u(x)|^{2} d x \\
\leq & C\left\{\int_{B\left(x_{0}, r\right)}\{m(x, V)\}^{n}|L u(x)|^{2} d x+\int_{B\left(x_{0}, r\right)} V(x)\{m(x, V)\}^{n}|u(x)|^{2} d x\right\} .
\end{aligned}
$$

The theorem follows by integrating above inequality in $x_{0}$ over $\mathbb{R}^{n}$ and observing that

$$
\int_{\left|x-x_{0}\right|<\frac{1}{m\left(x_{0}, V\right)}} d x_{0} \approx\{m(x, V)\}^{-n}
$$

which is a consequence of part (b) of Lemma 1.5. 


\section{The Fefferman-Phong Estimate}

In this section we establish a Fefferman-Phong type estimate for the Schrödinger operator with magnetic field

$$
H=\sum_{j=1}^{n}\left(\frac{1}{i} \frac{\partial}{\partial x_{j}}-a_{j}\right)^{2}+V
$$

under the assumption that $|\operatorname{curl} \vec{a}|+V+1 \in(R H)_{\frac{n}{2}}$, loc (Theorem 2.7).

We begin with a partition of unity.

The following proposition follows easily from [Hö, Theorem 1.4.10, p. 30] and part (b) of Lemma 1.5.

Proposition 2.1. Suppose $W \in(R H)_{n / 2}$, loc and $W \geq 1$ a.e. Then there exist a sequence $\left\{x_{\ell}\right\}$ in $\mathbb{R}^{n}$ and $\phi_{\ell} \in C_{0}^{\infty}\left(\mathbb{R}^{n}\right)$ such that

(a) $\mathbb{R}^{n}=\bigcup_{\ell=1}^{\infty} B_{\ell}$ where $B_{\ell}=B\left(x_{\ell}, \frac{1}{m\left(x_{\ell}, W\right)}\right)$,

(b) $\phi_{\ell} \in C_{0}^{\infty}\left(B_{\ell}\right)$ and $\sum_{\ell=1}^{\infty} \phi_{\ell} \equiv 1$ on $\mathbb{R}^{n}$,

(c) $\left|\partial_{x}^{\beta} \phi_{\ell}(x)\right| \leq C\{m(x, W)\}^{|\beta|}$ for $|\beta| \leq 2$,

(d) $\sum_{\ell=1}^{\infty} \chi_{B_{\ell}}(x) \leq C$ where $\chi_{B_{\ell}}$ denotes the characteristic function of $B_{\ell}$.

Recall that $L_{j}=\frac{1}{i} \frac{\partial}{\partial x_{j}}-a_{j}(x), 1 \leq j \leq n$. It is easy to see that, for $1 \leq j, k \leq$ $n$,

$$
\left[L_{j}, L_{k}\right]=L_{j} L_{k}-L_{k} L_{j}=\frac{1}{i}\left\{\frac{\partial a_{j}}{\partial x_{k}}-\frac{\partial a_{k}}{\partial x_{j}}\right\}=\frac{1}{i} b_{j k}(x) .
$$

Let $\langle$,$\rangle denote the inner product on L^{2}\left(\mathbb{R}^{n}\right)$, i.e.,

$$
\langle u, v\rangle=\int_{\mathbb{R}^{n}} u \bar{v} d x .
$$

Let $\psi \in C^{\infty}\left(\mathbb{R}^{n}\right)$ such that $\psi \geq 1$ on $\mathbb{R}^{n}$. Since $\left\langle L_{j} u, v\right\rangle=\left\langle u, L_{j} v\right\rangle$, following [H, p. 84], we have

$$
\begin{aligned}
\left\|\psi^{-1 / 2} b_{j k} u\right\|_{2}^{2} & =\left\langle\psi^{-1 / 2}\left[L_{j}, L_{k}\right] u, \psi^{-1 / 2}\left[L_{j}, L_{k}\right] u\right\rangle \\
& =\left\langle\left(L_{j} L_{k}-L_{k} L_{j}\right) u, \psi^{-1}\left[L_{j}, L_{k}\right] u\right\rangle \\
& =\left\langle L_{k} u, L_{j}\left\{\psi^{-1}\left[L_{j}, L_{k}\right] u\right\}\right\rangle-\left\langle L_{j} u, L_{k}\left\{\psi^{-1}\left[L_{j}, L_{k}\right] u\right\}\right\rangle \\
& =\left\langle L_{k} u,\left[L_{j}, \psi^{-1}\left[L_{j}, L_{k}\right]\right] u\right\rangle+\left\langle L_{k} u, \psi^{-1}\left[L_{j}, L_{k}\right] L_{j} u\right\rangle \\
& -\left\langle L_{j} u,\left[L_{k}, \psi^{-1}\left[L_{j}, L_{k}\right]\right] u\right\rangle-\left\langle L_{j} u, \psi^{-1}\left[L_{j}, L_{k}\right] L_{k} u\right\rangle
\end{aligned}
$$

where $u \in C_{0}^{2}\left(\mathbb{R}^{n}\right)$. Note that

$$
\left|\left[L_{j}, \psi^{-1}\left[L_{j}, L_{k}\right]\right]\right|=\left|\frac{\partial}{\partial x_{j}}\left\{\psi^{-1} b_{j k}\right\}\right| \leq \frac{\left|\nabla b_{j k}\right|}{\psi}+\frac{\left|b_{j k}\right||\nabla \psi|}{\psi^{2}}
$$

and

$$
\left|\psi^{-1}\left[L_{j}, L_{k}\right]\right|=\left|\psi^{-1} b_{j k}\right|
$$


We are now ready to prove

Theorem 2.7. Suppose $\vec{a}(x) \in C^{2}\left(\mathbb{R}^{n}\right), V \in L_{\mathrm{loc}}^{n / 2}\left(\mathbb{R}^{n}\right)$ and $V(x) \geq 0$ a.e. Also assume that

$$
\left\{\begin{array}{l}
|B(x)|+V(x)+1 \in(R H)_{\frac{n}{2},} \text { loc } \\
|B(x)| \leq C\{m(x,|B|+V+1)\}^{2} \\
|\nabla B(x)| \leq C\{m(x,|B|+V+1)\}^{3}
\end{array}\right.
$$

where $B(x)=\operatorname{curl} \vec{a}(x)=\left(b_{j k}(x)\right)_{1 \leq j, k \leq n}$. Then, for $u \in C_{0}^{1}\left(\mathbb{R}^{n}\right)$,

$$
\begin{array}{rl}
\int_{\mathbb{R}^{n}} & \left.m(x,|B|+V+1) u(x)\right|^{2} d x \\
\leq C\left\{\sum_{j=1}^{n} \int_{\mathbb{R}^{n}}\left|\left(\frac{1}{i} \frac{\partial}{\partial x_{j}}-a_{j}(x)\right) u\right|^{2} d x+\int_{\mathbb{R}^{n}}(V(x)+1)|u|^{2} d x\right\} .
\end{array}
$$

Proof. Since $W=|B|+V+1 \in(R H)_{n / 2}$, loc, we may apply Proposition 2.1 to obtain $\left\{x_{\ell}\right\} \subset \mathbb{R}^{n}$ and $\left\{\phi_{\ell}\right\} \subset C_{0}^{\infty}\left(\mathbb{R}^{n}\right)$ which satisfy (a)-(d) in the proposition.

Let

$$
\psi(x)=\sum_{\ell=1}^{\infty}\left\{m\left(x_{\ell},|B|+V+1\right)\right\}^{2} \phi_{\ell}(x) .
$$

Then $\psi \in C^{\infty}\left(\mathbb{R}^{n}\right)$,

$$
\left\{\begin{array}{l}
\psi(x) \approx\{m(x,|B|+V+1)\}^{2}, \\
|\nabla \psi(x)| \leq C\{m(x,|B|+V+1)\}^{3} .
\end{array}\right.
$$

Thus, by assumption (2.8),

$$
|B(x)| \leq C \psi(x) \text { and }|\nabla B(x)| \leq C \psi^{3 / 2}(x) .
$$

We now use formula (2.4). Note that, by (2.5), (2.6), (2.10) and (2.11),

$$
\mid\left[L_{j}, \psi^{-1}\left[L_{j}, L_{k}\right] \mid \leq C \psi^{1 / 2}(x), \quad 1 \leq j, k \leq n,\right.
$$

and

$$
\left|\psi^{-1}\left[L_{k}, L_{j}\right]\right| \leq C
$$

It follows that

$$
\int_{\mathbb{R}^{n}}\left\{\psi^{-1 / 2}|B| u\right\}^{2} d x \leq C\left\{\int_{\mathbb{R}^{n}}|L u|^{2} d x+\int_{\mathbb{R}^{n}}\left|L u \| \psi^{1 / 2} u\right| d x\right\}
$$

where

$$
|L u|^{2}=\sum_{j=1}^{n}\left|L_{j} u\right|^{2}=\sum_{j=1}^{n}\left|\left(\frac{1}{i} \frac{\partial}{\partial x_{j}}-a_{j}(x)\right) u\right|^{2} .
$$

Thus,

$$
\int_{\mathbb{R}^{n}}\left\{\frac{|B|^{2}}{\psi}+V\right\}|u|^{2} d x \leq C\left\{\int_{\mathbb{R}^{n}}|L u|^{2} d x+\int_{\mathbb{R}^{n}}|L u|\left|\psi^{1 / 2} u\right| d x+\int_{\mathbb{R}^{n}} V|u|^{2} d x\right\} .
$$

We claim that

$$
\int_{\mathbb{R}^{n}} \psi|u|^{2} d x \leq C\left\{\int_{\mathbb{R}^{n}}|L u|^{2} d x+\int_{\mathbb{R}^{n}}\left\{\frac{|B|^{2}}{\psi}+V+1\right\}|u|^{2} d x\right\} .
$$


The theorem follows easily from (2.12) and (2.13).

To see (2.13), we note that there exists $c>0$, such that, if $x \in \mathbb{R}^{n}, r=$ $\{m(x,|B|+V+1)\}^{-1}$, then

$$
\left|\left\{y \in B(x, r): \frac{|B(y)|^{2}}{\psi(y)}+V(y)+1 \geq \frac{c}{r^{2}}\right\}\right| \geq c r^{n} .
$$

Indeed, since $\psi(y) \sim r^{-2}$ for $y \in B(x, r)$,

$$
\begin{aligned}
\{y \in B(x, r): & \left.|B(y)|+V(y)+1 \geq \frac{c_{0}}{r^{2}}\right\} \\
& \subset\left\{y \in B(x, r): \frac{|B(y)|^{2}}{\psi(y)}+V(y)+1 \geq \frac{c}{r^{2}}\right\}
\end{aligned}
$$

where $c>0$ depends on $c_{0}>0$. Thus, by Proposition 1.4,

$$
\begin{aligned}
\mid\{y \in & \left.B(x, r): \frac{|B(y)|^{2}}{\psi(y)}+V(y)+1 \geq \frac{c}{r^{2}}\right\} \mid \\
& \geq\left|\left\{y \in B(x, r):|B(y)|+V(y)+1 \geq \frac{c_{0}}{r^{2}}\right\}\right| \\
& \geq c r^{n} .
\end{aligned}
$$

This proves (2.14).

Now, let $r=r_{\ell}=\left\{m\left(x_{\ell},|B|+V+1\right)\right\}^{-1}$; by Lemma 1.8 and (2.14),

$$
\int_{B_{\ell}} \psi(y)|u(y)|^{2} d y \leq C\left\{\int_{B_{\ell}}|L u|^{2} d y+\int_{B_{\ell}}\left\{\frac{|B(y)|^{2}}{\psi(y)}+V(y)+1\right\}|u(y)|^{2} d y\right\}
$$

where $B_{\ell}=B\left(x_{\ell}, r_{\ell}\right), \ell=1,2, \ldots$

Since

$$
1 \leq \sum_{\ell=1}^{\infty} \chi_{B_{\ell}}(y) \leq C \quad \text { for any } y \in \mathbb{R}^{n},
$$

(2.13) follows by summing (2.15) over $\ell=1,2, \ldots$

This completes the proof of Theorem 2.7 .

\section{Eigenvalue Asymptotics}

This section is devoted to the proof of Theorem 0.9 stated in the introduction.

We begin with a lower bound for $N(\lambda, H(\vec{a}, V))$.

Lemma 3.1. Suppose $\vec{a}(x) \in C^{2}\left(\mathbb{R}^{n}\right)$ and $V(x) \geq 0$. Also assume that

$$
\left\{\begin{array}{l}
|B|+V+1 \in(R H)_{\frac{n}{2}}, \text { loc } \\
|B(x)| \leq C\{m(x,|B|+V+1)\}^{2}
\end{array}\right.
$$

where $B=B(x)=\operatorname{curl} \vec{a}(x)$. Then there exists $c>0$ such that, for any $\lambda>0$,

$$
N(\lambda, H(\vec{a}, V)) \geq c \lambda^{n / 2}\left|\left\{x \in \mathbb{R}^{n}: m(x,|B|+V+1) \leq c \sqrt{\lambda}\right\}\right| .
$$

Proof. Fix $\lambda>0$. We divide $\mathbb{R}^{n}$ into a grid of cubes $\left\{Q_{\ell}\right\}$ with side length $1 / \sqrt{\lambda}$ whose sides are parallel to the coordinate axes. Let

$$
E_{\lambda}=\left\{x \in \mathbb{R}^{n}: m(x,|B|+V+1) \leq \sqrt{\lambda}\right\} .
$$


Then

$$
\left|E_{\lambda}\right|=\sum_{\ell}\left|Q_{\ell} \cap E_{\lambda}\right| \leq\left(\frac{1}{\lambda}\right)^{\frac{n}{2}} \cdot m
$$

where $m$ is the number of cubes $Q_{\ell}$ such that $Q_{\ell} \cap E_{\lambda} \neq \emptyset$.

We shall show that there exists a $m$-dimensional subspace $\mathcal{H}$ of $L^{2}\left(\mathbb{R}^{n}\right)$ such that

$$
\sum_{j=1}^{n}\left\langle L_{j} u, L_{j} u\right\rangle+\langle V u, u\rangle \leq C \lambda\|u\|_{2}^{2}
$$

for every $u$ in $\mathcal{H}$. This, together with the minimax principle, implies that

$$
\begin{aligned}
N(C \lambda, H(\vec{a}, V)) & \geq m \geq \lambda^{\frac{n}{2}}\left|E_{\lambda}\right| \\
& =\lambda^{\frac{n}{2}}\left|\left\{x \in \mathbb{R}^{n}: m(x,|B|+V+1) \leq \sqrt{\lambda}\right\}\right| .
\end{aligned}
$$

The lemma then follows since $\lambda$ is arbitrary.

To show (3.3),we suppose $Q_{\ell} \cap E_{\lambda} \neq \emptyset$ for $\ell=1,2, \ldots, m$. We define, for $x, y \in Q_{\ell}$,

$$
g_{j}^{\ell}(x, y)=\sum_{k=1}^{n}\left(x_{k}-y_{k}\right) \int_{0}^{1}\left(\frac{\partial a_{j}}{\partial x_{k}}-\frac{\partial a_{k}}{\partial x_{j}}\right)(y+t(x-y)) t d t
$$

where $x=\left(x_{1}, x_{2}, \ldots, x_{n}\right), y=\left(y_{1}, \ldots, y_{n}\right)$, and

$$
h_{j}^{\ell}(x)=\frac{1}{\left|Q_{\ell}\right|} \int_{Q_{\ell}} g_{j}^{\ell}(x, y) d y, \quad \ell=1,2, \ldots, m .
$$

(See $[\mathrm{I}])$. It follows that

$$
\begin{aligned}
g_{j}^{\ell}(x, y)= & \int_{0}^{1} \frac{d}{d t}\left\{a_{j}(y+t(x-y))\right\} t d t \\
& \quad-\sum_{k=1}^{n}\left(x_{k}-y_{k}\right) \frac{\partial}{\partial x_{j}} \int_{0}^{1} a_{k}(y+t(x-y)) d t \\
= & a_{j}(x)-\frac{\partial}{\partial x_{j}}\left\{\sum_{k=1}^{n}\left(x_{k}-y_{k}\right) \int_{0}^{1} a_{k}(y+t(x-y)) d t\right\} .
\end{aligned}
$$

Let

$$
\Phi^{\ell}(x)=\frac{1}{\left|Q_{\ell}\right|} \int_{Q_{\ell}}\left\{\sum_{k=1}^{n}\left(x_{k}-y_{k}\right) \int_{0}^{1} a_{k}(y+t(x-y)) d t\right\} d y
$$

for $\ell=1,2, \ldots, m$.

We have

$$
h_{j}^{\ell}(x)=a_{j}(x)-\frac{\partial}{\partial x_{j}} \Phi^{\ell}(x)
$$

for $x \in Q_{\ell}, \ell=1,2, \ldots, m$. Hence,

$$
\left|\left(\frac{1}{i} \frac{\partial}{\partial x_{j}}-a_{j}(x)\right) u\right|=\left|\left(\frac{1}{i} \frac{\partial}{\partial x_{j}}-h_{j}^{\ell}(x)\right)\left(e^{-i \Phi^{\ell}} u\right)\right| \quad \text { on } Q_{\ell} .
$$


Now, let $\eta \in C_{0}^{\infty}(Q(0,1))$ with $\|\eta\|_{2}=1$ where $Q(x, r)$ denotes the cube centered at $x$ with side length $r$. Suppose $Q_{\ell}=Q\left(x_{\ell}, \frac{1}{\sqrt{\lambda}}\right), \ell=1,2, \ldots, m$. We let

$$
\eta_{\ell}(x)=\lambda^{n / 4} \eta\left(\sqrt{\lambda}\left(x-x_{\ell}\right)\right)
$$

and

$$
\mathcal{H}=\operatorname{span}\left\{\eta_{\ell}(x) e^{i \Phi^{\ell}(x)}: \ell=1,2, \ldots, m\right\} .
$$

Clearly, $\operatorname{dim} \mathcal{H}=m$ and

$$
\begin{aligned}
\int_{\mathbb{R}^{n}} & \left|\left(\frac{1}{i} \frac{\partial}{\partial x_{j}}-a_{j}(x)\right)\left(\eta_{\ell} e^{i \Phi^{\ell}}\right)\right|^{2} d x \\
& =\int_{Q_{\ell}}\left|\left(\frac{1}{i} \frac{\partial}{\partial x_{j}}-h_{j}^{\ell}(x)\right)\left(\eta_{\ell}\right)\right|^{2} d x \\
& \leq \int_{Q_{\ell}}\left|\nabla \eta_{\ell}\right|^{2} d x+C \lambda^{\frac{n}{2}} \int_{Q_{\ell}}\left|h_{j}^{\ell}(x)\right|^{2} d x \\
& \leq C \lambda+C \lambda^{\frac{n}{2}} \int_{Q_{\ell}}\left|h_{j}^{\ell}(x)\right|^{2} d x .
\end{aligned}
$$

We claim that, for $1 \leq j \leq n$ and $1 \leq \ell \leq m$,

$$
\int_{Q_{\ell}}\left|h_{j}^{\ell}(x)\right|^{2} d x \leq C \lambda^{-(n / 2)+1} .
$$

In fact, since $Q_{\ell} \cap E_{\lambda} \neq \emptyset$,

$$
\inf _{x \in Q_{\ell}} m(x,|B|+V+1) \leq \sqrt{\lambda} .
$$

By part (b) of Lemma 1.5,

$$
\sup _{x \in Q_{\ell}} m(x,|B|+V+1) \leq C \sqrt{\lambda} .
$$

Thus, for $x, y \in Q_{\ell}$,

$$
\left|g_{j}^{\ell}(x, y)\right| \leq C \sqrt{\lambda}
$$

where we have used assumption (3.2). (3.10) then follows from (3.5).

To summarize, we have proved that

$$
\sum_{j=1}^{n} \int_{\mathbb{R}^{n}}\left|\left(\frac{1}{i} \frac{\partial}{\partial x_{j}}-a_{j}(x)\right)\left(\eta_{\ell} e^{i \Phi^{\ell}}\right)\right|^{2} d x \leq C \lambda, \quad \ell=1,2, \ldots, m .
$$

Finally, note that, since

$$
\frac{1}{\sqrt{\lambda}} \leq \frac{C}{m\left(x_{\ell},|B|+V+1\right)}, \quad \ell=1,2, \ldots, m,
$$


by (1.6),

$$
\begin{aligned}
& \int_{\mathbb{R}^{n}} V\left|\eta_{\ell} e^{i \Phi^{\ell}}\right|^{2} d x \leq C \lambda^{\frac{n}{2}} \int_{Q\left(x_{\ell}, \frac{1}{\sqrt{\lambda}}\right)} V(x) d x \\
& \quad=C \lambda \cdot \frac{\left(\frac{1}{\sqrt{\lambda}}\right)^{2}}{\left|Q\left(x_{\ell}, \frac{1}{\sqrt{\lambda}}\right)\right|} \int_{Q\left(x_{\ell}, \frac{1}{\sqrt{\lambda}}\right)} V(x) d x \leq C \lambda \cdot \frac{r_{\ell}^{2}}{\left|B\left(x_{\ell}, C r_{\ell}\right)\right|^{2}} \int_{B\left(x_{\ell}, C r_{\ell}\right)} V(x) d x \\
& \quad \leq C \lambda
\end{aligned}
$$

where $r_{\ell}=\left\{m\left(x_{\ell},|B|+V+1\right)\right\}^{-1}$.

Thus,

$$
\sum_{j=1}^{n}\left\langle L_{j} u, L_{j} u\right\rangle+\langle V u, u\rangle \leq C \lambda\|u\|_{2}^{2}
$$

if $u=\eta_{\ell} e^{i \Phi^{\ell}}, \ell=1,2, \ldots, m$. (3.3) is then proved. The proof of Lemma 3.1 is finished.

We now establish an upper bound for $N(\lambda, H(\vec{a}, V))$, using the estimate in Theorem 2.7.

Lemma 3.14. Suppose $\vec{a} \in C^{2}\left(\mathbb{R}^{n}\right)$ and $V(x) \geq 0$. Also assume that

$$
\left\{\begin{array}{l}
|B|+V+1 \in(R H)_{\frac{n}{2}}, \text { loc } \\
|B(x)| \leq C\{m(x,|B|+V+1)\}^{2} \\
|\nabla B(x)| \leq C\{m(x,|B|+V+1)\}^{3}
\end{array}\right.
$$

where $B=\operatorname{curl} \vec{a}$. Then there exists $C>0$ such that, for any $\lambda \geq C$,

$$
N(\lambda, H(\vec{a}, V)) \leq C \lambda^{n / 2}\left|\left\{x \in \mathbb{R}^{n}: m(x,|B|+V+1) \leq C \sqrt{\lambda}\right\}\right| .
$$

Proof. Let

$$
E_{\lambda}=\left\{x \in \mathbb{R}^{n}: m(x,|B|+V+1) \leq \sqrt{\lambda}\right\} .
$$

By the minimax principle, it suffices to show that, there exists a subspace $\mathcal{H}$ of $L^{2}\left(\mathbb{R}^{n}\right)$ such that

$$
\operatorname{dim} \mathcal{H} \leq C \lambda^{n / 2}\left|E_{\lambda}\right|
$$

and for any $u \perp \mathcal{H}$ and $\lambda \geq C$,

$$
\sum_{j=1}^{n}\left\langle L_{j} u, L_{j} u\right\rangle+\langle V u, u\rangle \geq c \lambda\|u\|_{2}^{2} .
$$

To this end, let $\left\{\phi_{\ell}\right\}_{\ell=1}^{\infty}$ be the sequence of functions constructed in Proposition 2.1 for the potential $W=|B(x)|+V(x)+1$. Then

$$
\int_{\mathbb{R}^{n}}\left|L_{j}\left(u \phi_{\ell}\right)\right|^{2} d x \leq 2\left\{\int_{\mathbb{R}^{n}}\left|L_{j}(u)\right|^{2} \phi_{\ell}^{2} d x+\int_{\mathbb{R}^{n}}\left|\nabla \phi_{\ell}\right|^{2}|u|^{2} d x\right\} .
$$


It follows from $\left|\nabla \phi_{\ell}(x)\right| \leq C\{m(x,|B|+V+1)\}$ that

$$
\begin{aligned}
& \int_{\mathbb{R}^{n}}\left|L_{j}\left(u \phi_{\ell}\right)\right|^{2} d x+\int_{\mathbb{R}^{n}} V\left|u \phi_{\ell}\right|^{2} d x \\
& \leq C\left\{\int_{\mathbb{R}^{n}}\left|L_{j}(u)\right|^{2} \phi_{\ell}^{2} d x+\int_{B_{\ell}}|m(x,|B|+V+1) u|^{2} d x+\int_{\mathbb{R}^{n}} V|u|^{2} \phi_{\ell}^{2} d x\right\}
\end{aligned}
$$

where $B_{\ell}=B\left(x_{\ell},\left\{m\left(x_{\ell},|B|+V+1\right)\right\}^{-1}\right), \ell=1,2, \ldots$

Summing over $\ell=1,2, \ldots$, we have

$$
\begin{aligned}
& \sum_{\ell=1}^{\infty}\left\{\int_{\mathbb{R}^{n}}\left|L_{j}\left(u \phi_{\ell}\right)\right|^{2} d x+\int_{\mathbb{R}^{n}} V\left|u \phi_{\ell}\right|^{2} d x\right\} \\
& \leq C\left\{\int_{\mathbb{R}^{n}}\left|L_{j}(u)\right|^{2} d x+\int_{\mathbb{R}^{n}}|m(x,|B|+V+1) u|^{2} d x+\int_{\mathbb{R}^{n}} V|u|^{2} d x\right\} \\
& \quad \leq C\left\{\int_{\mathbb{R}^{n}}|L(u)|^{2} d x+\int_{\mathbb{R}^{n}}(V+1)|u|^{2} d x\right\}
\end{aligned}
$$

where we have used Theorem 2.7 in the last inequality.

We need to consider two cases for $\ell$.

First, if $B_{\ell} \cap E_{\lambda}^{c} \neq \emptyset$, then

$$
\sup _{x \in B_{\ell}}\{m(x,|B|+V+1)\} \geq \sqrt{\lambda} .
$$

This, by part (b) of Lemma 1.5, implies that

$$
\inf _{x \in B_{\ell}}\{m(x,|B|+V+1)\} \geq c \sqrt{\lambda}
$$

It then follows from Theorem 2.7 that

$$
\begin{aligned}
\int_{\mathbb{R}^{n}}\left|L\left(u \phi_{\ell}\right)\right|^{2} d x+ & \int_{\mathbb{R}^{n}}(V+1)\left|u \phi_{\ell}\right|^{2} d x \\
& \geq c \int_{\mathbb{R}^{n}}\{m(x,|B|+V+1)\}^{2}\left|u(x) \phi_{\ell}\right|^{2} d x \\
& \geq c \lambda \int_{\mathbb{R}^{n}}\left|u \phi_{\ell}\right|^{2} d x
\end{aligned}
$$

Next, we consider the case $B_{\ell} \subset E_{\lambda}$. In this case we define the function $g_{j}^{\ell}(x, y)$ by (3.4) for $x, y \in B_{\ell}$ and $j=1,2, \ldots, n$. Then

$$
\begin{aligned}
\left|g_{j}^{\ell}(x, y)\right| \leq & \operatorname{diam}\left(B_{\ell}\right) \cdot \sup _{z \in B_{\ell}}|B(z)| \\
& \leq C \operatorname{diam}\left(B_{\ell}\right) \cdot \sup _{z \in B_{\ell}}\{m(z,|B|+V+1)\}^{2} \\
& \leq C m\left(x_{\ell},|B|+V+1\right)
\end{aligned}
$$

where we have used assumption (3.15) in the second inequality and part (b) of Lemma 1.5 in the third.

Now, let

$$
h_{j}^{\ell}(x)=\frac{1}{\left|B_{\ell}\right|} \int_{B_{\ell}} g_{j}^{\ell}(x, y) d y \quad \text { for } x \in B_{\ell} .
$$

Then

$$
\left|h_{j}^{\ell}(x)\right| \leq C m\left(x_{\ell},|B|+V+1\right)
$$


and

$$
h_{j}^{\ell}(x)=a_{j}(x)-\frac{\partial}{\partial x_{j}} \Phi^{\ell}(x) \quad \text { on } B_{\ell}
$$

as in the proof of Lemma 3.1 (see (3.6)). It follows that

$$
\begin{aligned}
\int_{\mathbb{R}^{n}}\left|L_{j}\left(u \phi_{\ell}\right)\right|^{2} d x= & \int_{\mathbb{R}^{n}}\left|\left(\frac{1}{i} \frac{\partial}{\partial x_{j}}-h_{j}^{\ell}(x)\right)\left(u \phi_{\ell} e^{-i \Phi^{\ell}}\right)\right|^{2} d x \\
& \geq \int_{\mathbb{R}^{n}}\left|\frac{\partial}{\partial x_{j}}\left(u \phi_{\ell} e^{-i \Phi^{\ell}}\right)\right|^{2}-C \int_{\mathbb{R}^{n}}\{m(x,|B|+V+1)\}^{2}\left|u \phi_{\ell}\right|^{2} d x .
\end{aligned}
$$

Hence, by Theorem 2.7, if $B_{\ell} \subset E_{\lambda}$,

$$
\int_{B_{\ell}}\left|\nabla\left(u \phi_{\ell} e^{-i \Phi^{\ell}}\right)\right|^{2} \leq C\left\{\int_{\mathbb{R}^{n}}\left|L\left(u \phi_{\ell}\right)\right|^{2} d x+\int_{\mathbb{R}^{n}}(V+1)\left|u \phi_{\ell}\right|^{2} d x\right\} .
$$

To continue, for each $B_{\ell}=B\left(x_{\ell}, r_{\ell}\right) \subset E_{\lambda}$, let $Q_{\ell}=Q\left(x_{\ell}, 2 r_{\ell}\right)$. Then $B_{\ell} \subset Q_{\ell}$. We divide $Q_{\ell}$ further into a set of disjoint subcubes $\left\{Q_{\ell}^{\beta}\right\}$ with side length $\approx 1 / \sqrt{\lambda}$. This is possible since $r_{\ell}=\left\{m\left(x_{\ell},|B|+V+1\right)\right\}^{-1} \geq(\sqrt{\lambda})^{-1}$.

By the Poincaré inequality, if

$$
\int_{Q_{\ell}^{\beta}} u \phi_{\ell} e^{-i \Phi^{\ell}} d x=0 \quad \text { for every } Q_{\ell}^{\beta}
$$

then

$$
\begin{aligned}
\lambda \int_{\mathbb{R}^{n}}\left|u \phi_{\ell}\right|^{2} & =\sum_{\beta} \lambda \int_{Q_{\ell}^{\beta}}\left|u \phi_{\ell} e^{-i \Phi^{\ell}}\right|^{2} d x \\
& \leq C \sum_{\beta} \int_{Q_{\ell}^{\beta}}\left|\nabla\left(u \phi_{\ell} e^{-i \Phi^{\ell}}\right)\right|^{2} d x \\
& =C \int_{B_{\ell}}\left|\nabla\left(u \phi_{\ell} e^{-i \Phi^{\ell}}\right)\right|^{2} d x \\
& \leq C\left\{\int_{\mathbb{R}^{n}}\left|L\left(u \phi_{\ell}\right)\right|^{2} d x+\int_{\mathbb{R}^{n}}(V+1)\left|u \phi_{\ell}\right|^{2} d x\right\}
\end{aligned}
$$

where the last inequality is (3.21).

Thus, putting together two cases, we may conclude that, if $u$ satisfies (3.22),

$$
\int_{\mathbb{R}^{n}}\left|L\left(u \phi_{\ell}\right)\right|^{2} d x+\int_{\mathbb{R}^{n}}(V+1)\left|u \phi_{\ell}\right|^{2} d x \geq c \lambda \int_{\mathbb{R}^{n}}\left|u \phi_{\ell}\right|^{2}
$$

for $\ell=1,2, \ldots$ It then follows from (3.19) that

$$
\begin{aligned}
\int_{\mathbb{R}^{n}}|L u|^{2} d x+\int_{\mathbb{R}^{n}} V|u|^{2} d x & \geq(c \lambda-1) \int_{\mathbb{R}^{n}}|u|^{2} d x \\
& \geq c_{3} \lambda \int_{\mathbb{R}^{n}}|u|^{2} d x
\end{aligned}
$$

if $u$ satisfies (3.22) and $\lambda \geq c_{4}>0$.

Finally, let

$$
\mathcal{H}=\operatorname{span}\left\{\phi_{\ell}(x) e^{i \Phi^{\ell}(x)} \chi_{Q_{\ell}^{\beta}}(x): B_{\ell} \subset E\right\}
$$


It is easy to see that, for each $B_{\ell} \subset E$, the number of cubes in $\left\{Q_{\ell}^{\beta}\right\} \leq C \lambda^{n / 2}\left|B_{\ell}\right|$. Thus,

$$
\begin{aligned}
\operatorname{dim} \mathcal{H} & \leq \sum_{B_{\ell} \subset E} C \lambda^{n / 2}\left|B_{\ell}\right| \leq C \lambda^{n / 2}\left|E_{\lambda}\right| \\
& =C \lambda^{n / 2}\left|\left\{x \in \mathbb{R}^{n}: m(x,|B|+V+1) \leq \sqrt{\lambda}\right\}\right|,
\end{aligned}
$$

and for any $u \in$ Domain of $H(\vec{a}, V), u \perp \mathcal{H}$ and $\lambda \geq c_{4}>0,(3.18)$ holds.

The proof is finished.

Finally, we are in a position to give the

Proof of Theorem 0.9. Note that, by Lemma 3.1, for any $\lambda>0$,

$$
\begin{aligned}
& \left|\left\{(x, \xi) \in \mathbb{R}^{n} \times \mathbb{R}^{n}:|\xi|^{2}+\{C m(x,|B|+V+1)\}^{2}<\lambda\right\}\right| \\
& \quad=C_{n} \int_{\left\{x \in \mathbb{R}^{n}:\{C m(x,|B|+V+1)\}^{2}<\lambda\right\}}\left[\lambda-\{C m(x,|B|+V+1)\}^{2}\right]^{n / 2} d x \\
& \quad \leq C_{n} \lambda^{n / 2}\left|\left\{x \in \mathbb{R}^{n}: m(x,|B|+V+1)<\frac{\sqrt{\lambda}}{C}\right\}\right| \\
& \quad \leq C N(\lambda, H(\vec{a}, V)), \text { if } C \text { is large. }
\end{aligned}
$$

Similarly, by Lemma 3.14 , for $\lambda \geq C>0$,

$$
\begin{aligned}
& \left|\left\{(x, \xi) \in \mathbb{R}^{n} \times \mathbb{R}^{n}:|\xi|^{2}+\{c m(x,|B|+V+1)\}^{2}<\lambda\right\}\right| \\
& \quad=C_{n} \int_{\left\{x \in \mathbb{R}^{n}:\{c m(x,|B|+V+1)\}^{2}<\lambda\right\}}\left[\lambda-\{c m(x,|B|+V+1)\}^{2}\right]^{n / 2} d x \\
& \quad \geq C_{n} \lambda^{n / 2}\left|\left\{x \in \mathbb{R}^{n}: c m(x,|B|+V+1) \leq \frac{\sqrt{\lambda}}{2}\right\}\right| \\
& \quad \geq C N(\lambda, H(\vec{a}, V)) \text { if } c \text { is small. }
\end{aligned}
$$

This completes the proof of Theorem 0.9.

Remark 3.23. If $\lim _{|x| \rightarrow \infty} m(x,|B|+V+1)=\infty$, then

$$
\left|\left\{x \in \mathbb{R}^{n}: m(x,|B|+V+1)<\sqrt{\lambda}\right\}\right|
$$

is finite for any $\lambda>0$. This, by Theorem 0.9 , implies that $N(\lambda, H)<\infty$ for every $\lambda>0$. It follows that $H$ has a discrete spectrum.

Conversely, suppose $\lim _{|x| \rightarrow \infty} m(x,|B|+V+1) \neq \infty$. Then there exists a sequence $\left\{x_{\ell}\right\}$ such that $\left|x_{\ell}\right| \rightarrow \infty$ and $m\left(x_{\ell},|B|+V+1\right) \leq M$. It follows from part (b) of Lemma 1.5 that

$$
\bigcup_{\ell=1}^{\infty} B\left(x_{\ell}, \frac{1}{M}\right) \subset\left\{x \in \mathbb{R}^{n}: m(x,|B|+V+1) \leq C \cdot M\right\} .
$$

Hence, $\left|\left\{x \in \mathbb{R}^{n}: m(x,|B|+V+1) \leq C \cdot M\right\}\right|=\infty$ and the spectrum of $H$ can not be discrete by Theorem 0.9 .

This gives the proof of Corollary 0.10 in the introduction since $m(x,|B|+V+1) \approx$ $m(x,|B|+V)+1$. 


\section{Exponential Decay of Eigenfunctions}

In this section we give the proof of Theorem 0.20 stated in the introduction. We begin with some general properties of eigenfunctions.

Recall that

$$
L_{j}=\frac{1}{i} \frac{\partial}{\partial x_{j}}-a_{j}(x) \quad \text { and } \quad|L u|^{2}=\sum_{j=1}^{n}\left|L_{j} u\right|^{2} .
$$

The following is a Caccioppoli-type inequality.

Lemma 4.1. Suppose $\vec{a} \in L_{\mathrm{loc}}^{2}\left(\mathbb{R}^{n}\right), V(x) \geq 0$ and $V \in L_{\mathrm{loc}}^{1}\left(\mathbb{R}^{n}\right)$. Also assume that $u \in L^{2}\left(\mathbb{R}^{n}\right), H(\vec{a}, V) u \in L^{2}\left(\mathbb{R}^{n}\right)$ and

$$
H(\vec{a}, V) u=\lambda u, \lambda>0 .
$$

Then, for any $x \in \mathbb{R}^{n}, R>0$,

$$
\int_{B(x, R)}|L u|^{2} d y \leq \frac{C}{R^{2}} \int_{B(x, 2 R)}|u|^{2} d y+C \lambda \int_{B(x, 2 R)}|u|^{2} d y .
$$

The proof of Lemma 4.1 is similar to that of the usual Caccioppoli's inequality and hence omitted.

Lemma 4.2. Under the same assumptions of Lemma 4.1, we have, for every $x \in$ $\mathbb{R}^{n}$,

$$
|u(x)| \leq C\left(\frac{1}{|B(x, R)|} \int_{B(x, R)}|u(y)|^{2} d y\right)^{1 / 2}
$$

if $\lambda R^{2} \leq 1$.

Proof. Fix $x_{0} \in \mathbb{R}^{n}$. Let $\varphi \in C_{0}^{\infty}\left(B\left(x_{0}, R / 2\right)\right)$ such that $\varphi \equiv 1$ on $B\left(x_{0}, R / 4\right)$, and $\left|\partial_{x}^{\beta} \varphi(x)\right| \leq C R^{-|\beta|}$ for $|\beta| \leq 2$. Then

$$
H(\vec{a}, V)(u \varphi)=\lambda u \cdot \varphi+\frac{2}{i} L_{j}(u) \cdot \frac{\partial \varphi}{\partial x_{j}}-u \cdot \Delta \varphi .
$$

It follows from [LS, Lemma 6, p. 7] that

$$
\begin{aligned}
|u(x) \varphi(x)| & =\left|(H(\vec{a}, V)+\varepsilon)^{-1}\left((\lambda+\varepsilon) u \varphi+\frac{2}{i} L_{j}(u) \cdot \frac{\partial \varphi}{\partial x_{j}}-u \Delta \varphi\right)\right| \\
& \leq(-\Delta+\varepsilon)^{-1}\left|(\lambda+\varepsilon) u \varphi+\frac{2}{i} L_{j}(u) \cdot \frac{\partial \varphi}{\partial x_{j}}-u \Delta \varphi\right| \\
& \leq C_{n} \int_{\mathbb{R}^{n}} \frac{(\lambda+\varepsilon)|u \varphi|+2|L u||\nabla \varphi|+|u \Delta \varphi|}{|x-y|^{n-2}} d y
\end{aligned}
$$

for any $\varepsilon>0$. Now let $\varepsilon=\lambda$ and $x \in B\left(x_{0}, R / 8\right)$;

$$
\begin{aligned}
|u(x)| & \leq C \int_{\mathbb{R}^{n}} \frac{\lambda|u \varphi|}{|x-y|^{n-2}} d y+\frac{C}{R^{n-1}} \int_{B\left(x_{0}, \frac{R}{2}\right)}|L u| d y+\frac{C}{R^{n}} \int_{B\left(x_{0}, \frac{R}{2}\right)}|u| d y \\
& \leq C \int_{\mathbb{R}^{n}} \frac{\lambda|u \varphi|}{|x-y|^{n-2}} d y+C\left(\frac{1}{R^{n}} \int_{B\left(x_{0}, R\right)}|u|^{2} d y\right)^{1 / 2}
\end{aligned}
$$

where we have used Hölder inequality, Lemma 4.1 and the assumption $\lambda R^{2} \leq 1$. 
It then follows from Young's inequality that, if

$$
\frac{1}{q}=\frac{1}{r}+\frac{1}{p}-1 \text { and }(n-2) r<n,
$$

then

$$
\begin{aligned}
\|u\|_{L^{q}\left(B\left(x_{0}, \frac{R}{8}\right)\right)} \leq C \lambda & \cdot R^{-n\left(1-\frac{1}{r}\right)+2}\|u\|_{L^{p}\left(B\left(x_{0}, \frac{R}{2}\right)\right)} \\
& +C R^{\frac{n}{q}}\left(\frac{1}{R^{n}} \int_{B\left(x_{0}, R\right)}|u|^{2} d y\right)^{1 / 2} .
\end{aligned}
$$

Thus,

$$
\begin{aligned}
& \left(\frac{1}{R^{n}} \int_{B\left(x_{0}, \frac{R}{8}\right)}|u|^{q} d y\right)^{1 / q} \\
& \quad \leq C\left\{\left(\frac{1}{R^{n}} \int_{B\left(x_{0}, \frac{R}{2}\right)}|u|^{p} d y\right)^{1 / p}+\left(\frac{1}{R^{n}} \int_{B\left(x_{0}, R\right)}|u|^{2} d y\right)^{1 / 2}\right\}
\end{aligned}
$$

for any $p, q$ such that $2 \leq p \leq q \leq \infty$ and $(1 / q)-(1 / p)>-(2 / n)$.

The lemma then follows from above estimate by a bootstrap argument.

In the rest of this section we will assume that

$$
|B(x)|+V(x)+1 \in(R H)_{\frac{n}{2}}, \text { loc. }
$$

Definition 4.3. We define

$$
d(x, y)=\inf \left\{\int_{0}^{1} m(\gamma(s),|B|+V+1)\left|\gamma^{\prime}(s)\right| d s: \gamma(0)=x, \gamma(1)=y\right\} .
$$

Let

$$
E_{\lambda}=\left\{x \in \mathbb{R}^{n}: m(x,|B|+V+1) \leq \sqrt{\lambda}\right\}
$$

and

$$
d_{\lambda}(x)=\inf \left\{d(x, y): y \in E_{\lambda}\right\} .
$$

We first need to regularize the distance function $d_{\lambda}(x)$, as in [HN2].

Lemma 4.6. There exists $\psi_{\lambda} \in C^{\infty}\left(\mathbb{R}^{n}\right)$ such that

(a) $\left|d_{\lambda}(x)-\psi_{\lambda}(x)\right| \leq C$ for any $x \in \mathbb{R}^{n}$,

(b) $\left|\partial_{x}^{\beta} \psi_{\lambda}(x)\right| \leq C\{m(x,|B|+V+1)\}^{|\beta|}$ if $|\beta| \leq 2$ and $\beta \neq(0,0, \ldots, 0)$.

Proof. Let

$$
\psi_{\lambda}(x)=\sum_{\ell=1}^{\infty} d_{\lambda}\left(x_{\ell}\right) \phi_{\ell}(x)
$$

where $\left\{x_{\ell}\right\} \subset \mathbb{R}^{n}$ and $\left\{\phi_{\ell}\right\} \subset C_{0}^{\infty}\left(\mathbb{R}^{n}\right)$ are constructed in Proposition 2.1 using the potential $W=|B|+V+1$. It follows that

$$
\begin{aligned}
\left|d_{\lambda}(x)-\psi_{\lambda}(x)\right| & \leq \sum_{\ell}\left|d_{\lambda}\left(x_{\ell}\right)-d_{\lambda}(x)\right| \phi_{\ell}(x) \\
& \leq \sum_{\ell} d\left(x_{\ell}, x\right) \phi_{\ell}(x) .
\end{aligned}
$$


Since

$$
d(x, y) \leq C m(x,|B|+V+1)|x-y| \quad \text { if }|x-y|<\frac{1}{m(x,|B|+V+1)},
$$

we have

$$
\left|d_{\lambda}(x)-\psi_{\lambda}(x)\right| \leq C \quad \text { for any } x \in \mathbb{R}^{n} .
$$

To see part (b), let $\beta \neq(0, \ldots, 0)$ and $|\beta| \leq 2$. Then, if $x \in B\left(x_{\ell_{0}}, r_{\ell_{0}}\right)$ and $r_{\ell_{0}}=\left\{m\left(x_{\ell_{0}},|B|+V+1\right)\right\}^{-1}$,

$$
\begin{aligned}
\partial_{x}^{\beta} \psi_{\lambda}(x) & =\sum_{\ell} d_{\lambda}\left(x_{\ell}\right) \partial_{x}^{\beta} \phi_{\ell}(x) \\
& =\sum_{\ell}\left\{d_{\lambda}\left(x_{\ell}\right)-d_{\lambda}\left(x_{\ell_{0}}\right)\right\} \partial_{x}^{\beta} \phi_{\ell}(x) .
\end{aligned}
$$

Hence,

$$
\left|\partial_{x}^{\beta} \psi_{\lambda}(x)\right| \leq C \sum_{\ell}\left|\partial_{x}^{\beta} \phi_{\ell}(x)\right| \leq C\{m(x,|B|+V+1)\}^{|\beta|} .
$$

Remark 4.9. For some technique reasons, we shall need a sequence of bounded $C^{\infty}$ functions which approximate $\psi_{\lambda}(x)$. As in [HN2], we fix $F \in C^{\infty}(0, \infty)$ such that $F(t)=t$ for $t \in(0,1 / 2), F(t)=1$ if $t \geq 2$ and $0 \leq F(t) \leq t$ for all $t>0$. Let

$$
\psi_{\lambda, \mu}(x)=\mu F\left(\frac{\psi_{\lambda}(x)}{\mu}\right) \text { for } \mu \geq 1 .
$$

Then

$$
\begin{aligned}
& \psi_{\lambda, \mu}(x) \rightarrow \psi_{\lambda}(x) \quad \text { as } \mu \rightarrow \infty \text { for any fixed } x \in \mathbb{R}^{n} \text { and } \lambda>0, \\
& \psi_{\lambda, \mu}(x) \leq \psi_{\lambda}(x) \leq C \text { if } x \in E_{\lambda}, \\
& \left.\left|\partial_{x}^{\beta} \psi_{\lambda, \mu}(x)\right| \leq C\{m,|B|+V+1)\right\}^{|\beta|} \text { for }|\beta| \leq 2, \beta \neq(0,0, \ldots, 0) .
\end{aligned}
$$

Clearly, $\psi_{\lambda, \mu}$ is a bounded function of $x$.

We now establish an $L^{2}$ decay estimate for eigenfunctions of $H(\vec{a}, V)$.

Lemma 4.14. Suppose $\vec{a} \in C^{2}\left(\mathbb{R}^{n}\right)$ and $V \geq 0$. Also assume that

$$
\left\{\begin{array}{l}
|B|+V+1 \in(R H)_{\frac{n}{2}, \text { loc }}, \\
|B(x)| \leq C\{m(x,|B|+V+1)\}^{2}, \\
|\nabla B(x)| \leq C\{m(x,|B|+V+1)\}^{3} .
\end{array}\right.
$$

Then there exist $\varepsilon>0$ and $C>0$, such that, if $u \in L^{2}\left(\mathbb{R}^{n}\right), H(\vec{a}, V) u \in L^{2}\left(\mathbb{R}^{n}\right)$ and

$$
H(\vec{a}, V) u=\lambda u, \quad \lambda \geq 1,
$$

then

$$
\left\|e^{\varepsilon d_{C \lambda}} u\right\|_{2} \leq C\|u\|_{2} .
$$

Proof. Let $\psi=\psi_{T \lambda, \mu}(x)$ be given by (4.10) where $T>1$ is to be chosen later. A computation shows

$$
H(\vec{a}, V)\left(u e^{\varepsilon \psi}\right)=H(\vec{a}, V) u \cdot e^{\varepsilon \psi}+\frac{2}{i}\left(\frac{1}{i} \frac{\partial}{\partial x_{j}}-a_{j}\right) u \cdot \frac{\partial}{\partial x_{j}}\left(e^{\varepsilon \psi}\right)-u \Delta\left(e^{\varepsilon \psi}\right) .
$$


It follows that

$$
\begin{aligned}
& \int_{\mathbb{R}^{n}}\left|\left(\frac{1}{i} \frac{\partial}{\partial x_{j}}-a_{j}\right)\left(u e^{\varepsilon \psi}\right)\right|^{2} d x+\int_{\mathbb{R}^{n}} V\left|u e^{\varepsilon \psi}\right|^{2} d x \\
& =\left\langle H(\vec{a}, V)\left(u e^{\varepsilon \psi}\right), u e^{\varepsilon \psi}\right\rangle \\
& =\left\langle H(\vec{a}, V) u \cdot e^{\varepsilon \psi}, u e^{\varepsilon \psi}\right\rangle+\left\langle\frac{2}{i}\left(\frac{1}{i} \frac{\partial}{\partial x_{j}}-a_{j}\right) u \cdot \frac{\partial}{\partial x_{j}}\left(e^{\varepsilon \psi}\right), u e^{\varepsilon \psi}\right\rangle \\
& \quad-\left\langle u \cdot \Delta\left(e^{\varepsilon \psi}\right), u e^{\varepsilon \psi}\right\rangle .
\end{aligned}
$$

By (4.13),

$$
\begin{aligned}
& \left|\nabla\left(e^{\varepsilon \psi}\right)\right| \leq C \varepsilon\{m(x,|B|+V+1)\} e^{\varepsilon \psi}, \\
& \left|\Delta\left(e^{\varepsilon \psi}\right)\right| \leq C \varepsilon\{m(x,|B|+V+1)\}^{2} e^{\varepsilon \psi} .
\end{aligned}
$$

Thus, by Theorem 2.7, $\{m(x,|B|+V+1)\} u \in L^{2}\left(\mathbb{R}^{n}\right)$ and

$$
\begin{aligned}
\int_{\mathbb{R}^{n}} \mid & \left.\left(\frac{1}{i} \frac{\partial}{\partial x_{j}}-a_{j}\right)\left(u e^{\varepsilon \psi}\right)\right|^{2} d x+\int_{\mathbb{R}^{n}}\left|m(x,|B|+V+1) u e^{\varepsilon \psi}\right|^{2} d x \\
\leq & C \lambda \int_{\mathbb{R}^{n}}\left|u e^{\varepsilon \psi}\right|^{2} d x+C \varepsilon \int_{\mathbb{R}^{n}}\left|\left(\frac{1}{i} \frac{\partial}{\partial x_{j}}-a_{j}\right) u\right| \cdot m(x,|B|+V+1)|u| e^{2 \varepsilon \psi} d x \\
& +C \varepsilon \int_{\mathbb{R}^{n}}\left|m(x,|B|+V+1) u e^{\varepsilon \psi}\right|^{2}+C \int_{\mathbb{R}^{n}}\left|u e^{\varepsilon \psi}\right|^{2} d x \\
\leq & C \lambda \int_{\mathbb{R}^{n}}\left|u e^{\varepsilon \psi}\right|^{2} d x+C \varepsilon \int_{\mathbb{R}^{n}}\left|m(x,|B|+V+1) u e^{\varepsilon \psi}\right|^{2} d x \\
& +C \varepsilon \int_{\mathbb{R}^{n}}\left|\left(\frac{1}{i} \frac{\partial}{\partial x_{j}}-a_{j}\right)\left(u e^{\varepsilon \psi}\right)\right|^{2} d x
\end{aligned}
$$

where we have used the assumption $\lambda \geq 1$.

Choosing $\varepsilon$ so small that $C \varepsilon<1 / 2$, we obtain

$$
\int_{\mathbb{R}^{n}}\left|m(x,|B|+V+1) u e^{\varepsilon \psi}\right| d x^{2} \leq C \lambda \int_{\mathbb{R}^{n}}\left|u e^{\varepsilon \psi}\right|^{2} d x .
$$

Now, recall that $\psi(x)=\psi_{T \lambda, \mu}(x) \leq C$ if

We have

$$
x \in E_{T \lambda}=\left\{x \in \mathbb{R}^{n}: m(x,|B|+V+1) \leq \sqrt{T \lambda}\right\} .
$$

$$
\begin{aligned}
\int_{\mathbb{R}^{n}}\left|e^{\varepsilon \psi} u\right|^{2} d x & \leq C \int_{E_{T \lambda}}|u|^{2} d x+\frac{1}{T \lambda} \int_{\mathbb{R}^{n} \backslash E_{T \lambda}}\left|m(x,|B|+V+1) u e^{\varepsilon \psi}\right|^{2} d x \\
& \leq C \int_{\mathbb{R}^{n}}|u|^{2} d x+\frac{1}{T \lambda} \int_{\mathbb{R}^{n}}\left|m(x,|B|+V+1) u e^{\varepsilon \psi}\right|^{2} d x \\
& \leq C \int_{\mathbb{R}^{n}}|u|^{2} d x+\frac{C}{T} \int_{\mathbb{R}^{n}}\left|u e^{\varepsilon \psi}\right|^{2} d x
\end{aligned}
$$

where we have used (4.15) in the last inequality.

Choosing $T$ large, we get

$$
\int_{\mathbb{R}^{n}}\left|e^{\varepsilon \psi} u\right|^{2} d x \leq C \int_{\mathbb{R}^{n}}|u|^{2} d x .
$$

The lemma follows by letting $\mu \rightarrow \infty$ in $\psi=\psi_{T \lambda, \mu}(x)$ and using Fatou's Lemma, (4.11) and part (a) of Lemma 4.6. 
Lemma 4.16. Assume $\vec{a}(x)$ and $V(x)$ satisfy the same hypothesis of Lemma 4.14. Suppose $u \in L^{2}\left(\mathbb{R}^{n}\right)$ and $H(\vec{a}, V) u=\lambda u, \lambda \geq 1$. Then

$$
|u(x)| \leq C\{m(x,|B|+V+1)\}^{n / 2} e^{-\varepsilon d_{C \lambda}(x)}\|u\|_{2} .
$$

Proof. First, note that, by Lemma 4.2,

$$
|u(x)| \leq C \lambda^{\frac{n}{4}}\|u\|_{2} .
$$

Thus, we may assume $x \notin E_{C \lambda}$, i.e.

$$
m(x,|B|+V+1) \geq \sqrt{C \lambda} .
$$

Now let $r=\{m(x,|B|+V+1)\}^{-1}$. Note that, if $y \in B(x, r)$,

$$
\left|d_{C \lambda}(y)-d_{C \lambda}(x)\right| \leq d(x, y) \leq C .
$$

It follows from Lemma 4.2 that

$$
\begin{aligned}
|u(x)| & \leq C r^{-n / 2}\left\{\int_{B(x, r)}|u(y)|^{2} d y\right\}^{1 / 2} \\
& \leq C\{m(x,|B|+V+1)\}^{n / 2} e^{-\varepsilon d_{C \lambda}(x)}\left\{\int_{B(x, r)}\left|e^{\varepsilon d_{C \lambda}(y)} u(y)\right|^{2} d y\right\}^{1 / 2} \\
& \leq C\{m(x,|B|+V+1)\}^{n / 2} e^{-\varepsilon d_{C \lambda}(x)}\|u\|_{2}
\end{aligned}
$$

where we have used Lemma 4.14 in the last inequality.

We need one more lemma before we carry out the proof of Theorem 0.20.

Lemma 4.18. Suppose $W \in(R H)_{n / 2, \text { loc }}$ and $W \geq 1$ a.e. Then there exist $C>0$ and $k_{1}>0$ such that for any $x \in \mathbb{R}^{n}, y \in \mathbb{R}^{n}$,

$$
m(y, W) \leq C m(x, W)(1+d(x, y))^{k_{1}}
$$

where $d(x, y)$ is defined by (4.3).

Proof. We recall that, if $|x-y| \leq 1$, then

$$
\begin{aligned}
& m(y, W) \leq C m(x, W)(1+|x-y| m(x, W))^{k_{0}}, \\
& m(y, W) \geq \frac{c m(x, W)}{(1+|x-y| m(x, W))^{k_{0} /\left(k_{0}+1\right)}}
\end{aligned}
$$

for some $k_{0}>0$ (part (c) of Lemma 1.5).

We may assume that $|x-y| m(y, W) \geq 2$, for otherwise we have $m(x, W) \approx$ $m(y, W)$.

Let $\gamma:[0,1] \rightarrow \mathbb{R}^{n}$ be a curve such that $\gamma(0)=x$ and $\gamma(1)=y$ and

$$
\int_{0}^{1} m(\gamma(t), W)\left|\gamma^{\prime}(s)\right| d s \leq 2 d(x, y) .
$$

First we consider the case

$$
|\gamma(s)-y| \leq 1 \text { for any } s \in[0,1] .
$$


By (4.20) we have

$$
\begin{aligned}
& \int_{0}^{1} m(\gamma(s), W)\left|\gamma^{\prime}(s)\right| d s \geq c \int_{0}^{1} \frac{m(y, W)\left|\gamma^{\prime}(s)\right| d s}{(1+|\gamma(s)-y| m(y, W))^{k_{0} /\left(k_{0}+1\right)}} \\
& \geq c \cdot\{\text { the geodesic distance from } x \text { to } y \text { in } \\
& \left.\quad \text { the metric } \frac{m(y, W)}{(1+|z-y| m(y, W))^{k_{0} /\left(k_{0}+1\right)}} d z^{2}\right\} \\
& \geq c\{|x-y| m(y, W)\}^{\frac{1}{k_{0}+1}} .
\end{aligned}
$$

It follows that

$$
\begin{aligned}
m(y, W) & \leq C m(x, W)\{1+|x-y| m(y, W)\}^{k_{0} /\left(k_{0}+1\right)} \\
& \leq C m(x, W)\{1+d(x, y)\}^{k_{0}} .
\end{aligned}
$$

Next we consider the case

$$
\max _{0 \leq s \leq 1}|\gamma(s)-y|>1
$$

Let

$$
s_{0}=\sup \{s:|\gamma(s)-y|>1\} .
$$

As in the first case,

$$
\begin{aligned}
\int_{s_{0}}^{1} m(\gamma(s), W)\left|\gamma^{\prime}(s)\right| d s & \geq c\left\{\left|\gamma\left(s_{0}\right)-y\right| m(y, W)\right\}^{\frac{1}{k_{0}+1}} \\
& =c\{m(y, W)\}^{\frac{1}{k_{0}+1}}
\end{aligned}
$$

since $\left|\gamma\left(s_{0}\right)-y\right|=1$. Thus, since $m(x, W) \geq 1$,

$$
m(y, W) \leq C\{d(x, y)\}^{k_{0}+1} \leq C m(x, W)\{1+d(x, y)\}^{k_{0}+1} .
$$

The proof is complete.

Finally, we are in a position to give the

Proof of Theorem 0.20. Let $W=|B|+V+1$ and $E_{\lambda}=\left\{y \in \mathbb{R}^{n}: m(y, W) \leq \sqrt{\lambda}\right\}$. Suppose $x \in \mathbb{R}^{n}$ and $y \in E_{C \lambda}$. By Lemma 4.18

$$
\begin{aligned}
m(x, W) & \leq C m(y, W)\{1+d(x, y)\}^{k_{1}} \\
& \leq C \sqrt{\lambda}\{1+d(x, y)\}^{k_{1}} .
\end{aligned}
$$

It follows that

$$
m(x, W) \leq C \sqrt{\lambda}\left\{1+d_{C \lambda}(x)\right\}^{k_{1}} \leq C_{\varepsilon} \sqrt{\lambda} e^{\frac{\varepsilon}{2} d_{C \lambda}(x)}
$$

where $\varepsilon>0$ is given in Lemma 4.16 .

Now, by Lemma 4.16 ,

$$
\begin{aligned}
|u(x)| & \leq C\{m(x, W)\}^{n / 2} e^{-\varepsilon d_{C \lambda}(x)}\|u\|_{2} \\
& \leq C \lambda^{n / 4} e^{-\frac{\varepsilon}{2} d_{C \lambda}(x)}\|u\|_{2} .
\end{aligned}
$$

The proof of Theorem 0.20 is finished. 


\section{REFERENCES}

[A] S. Agmon, Lectures on Exponential Decay of Solutions of Second Order Elliptic Equations, Princeton Univ. Press, 1982. MR 85f:35019

[AHS] J. Avron, I. Herbst and B. Simon, Schrödinger operators with magnetic fields. I. General Interactions, Duke Math. J. 45(4) (1978), 847-883. MR 80k:35054

[F] C. Fefferman, The uncertainty principle, Bull. Amer. Math. Soc. 9 (1983), 129-206. MR 85f:35001

[Ge] F. Gehring, The $L^{p}$-integrability of the partial derivatives of a quasi-conformal mapping, Acta Math. 130 (1973), 265-277. MR 53:5861

[Gu] D. Gurarie, Nonclassical eigenvalue asymptotics for operators of Schrödinger type, Bull. Amer. Math. Soc. 15(2) (1986), 233-237. MR 88a:35177

[H] B. Helffer, Semi-Classical Analysis for the Schrödinger Operator and Applications, Lectures Notes in Math., vol. 1336, Springer-Verlag, 1988. MR 90c:81043

[HMo] B. Helffer and A. Mohamed, Caractérisation du spectre essentiel de l'opérateur de Schrödinger avec un champ magnétique, Ann. Inst. Fourier 38 (1988), 95-112. MR 90d:35215

[HN1] B. Helffer and J. Nourrigat, Hypoellipticité maximale pour des opérateurs polynômes de champs de vecteurs, Progress in Math. 58, Birkhäuser, Boston, 1985. MR 88i:35029

[HN2] - Decrossance a l'infini des fonctions propres de l'opérateur de Schrödinger avec champ electromagnétique polynomial, J. Anal. Math. 58 (1992), 263-275. MR 95e:35049

[Hö] L. Hörmander, The Analysis of Linear Partial Differential Operators I, Springer-Verlag, 1983. MR 85g:35002a

[I] A. Iwatsuka, Magnetic Schrödinger operators with compact resolvent, J. Math. Kyoto Univ. 26-3 (1986), 357-374. MR 87j:35287

[LMN] P. Lévy-Bruhl, A. Mohamed and J. Nourrigat, Etude spectrale d'opérateurs liés à des représentations de groupes nilpotents, J. Funct. Anal. 113 (1993), 65-93. MR 94k:22014

[LS] H. Leinfelder and C. Simader, Schrödinger operators with singular magnetic vector potentials, Math. Z. 176 (1981), 1-19. MR 82d:35073

[Ma] H. Matsumoto, Classical and non-classical eigenvalue asymptotics for magnetic Schrödinger operators, J. Funct. Anal. 95 (1991), 460-482. MR 92b:35114

$[\mathrm{MoN}] \quad$ A. Mohamed and J. Nourrigat, Encadrement du $N(\lambda)$ pour un opérator de Schrödinger avec un champ magnétique et un potentiel électrique, J. Math. Pures Appl. 70 (1991), 87-99. MR 92a:35122

$[\mathrm{MoR}]$ A. Mohamed and G. Raikov, On the spectral theory of the Schrödinger operator with electromagnetic potential, Pseudo-Differential Calculus and Mathematical Physics, Akademie Verlag, 1994, pp. 298-390. MR 96e:35122

$[\mathrm{Mu}]$ B. Muckenhoupt, Weighted norm inequality for the Hardy maximal function, Trans. Amer. Math. Soc. 165 (1972), 207-226. MR 45:2461

[R] D. Robert, Comportement asymptotique des valeurs propres d'opérateurs du type Schrödinger á potentiel "dégénéré", J. Math. Pures Appl. 61(9) (1982), 275-300. MR 84d:35117

[Sh1] Z. Shen, $L^{p}$ estimates for Schrödinger operators with certain potentials, Ann. Inst. Fourier 45(2) (1995), 513-546. CMP 95:16

[Sh2] - On the eigenvalue asymptotics of Schrödinger operators, preprint, 1995.

[Si1] B. Simon, Functional Integration and Quantum Physics, Academic Press, 1979. MR 84m:81066

[Si2] , Nonclassical eigenvalue asymptotics, J. Funct. Anal. 53 (1983), 84-98. MR 85i: 35113

[So] M. Solomjak, Spectral asymptotics of Schrödinger operators with non-regular homogeneous potential, Math. USSR Sb. 55 (1986), 19-38.

[St] E. Stein, Harmonic Analysis: Real-Variable Method, Orthogonality, and Oscillatory Integrals, Princeton Univ. Press, 1993. MR 95c:42002

[T] K. Tachizawa, Asymptotics distribution of eigenvalues of Schrödinger operators with nonclassical potentials, Tôhoku Math. J. 42 (1990), 381-406. MR 92a:35125

Department of Mathematics, University of Kentucky, Lexington, Kentucky 40506

E-mail address: shenz@ms.uky.edu 